\title{
Dynamics of a new stage-structured population model with transient and nontransient impulsive effects in a polluted environment
}

\author{
Qi Quan', Wenyan Tang', Jianjun Jiao ${ }^{1 *}$ (i) and Yuan Wang ${ }^{1}$
}

"Correspondence:

jiaojianjun05@126.com

${ }^{1}$ School of Mathematics and

Statistics, Guizhou University of Finance and Economics, Guiyang,

550025, P.R. China

\section{记) Springer}

\begin{abstract}
In this paper, we consider a new stage-structured population model with transient and nontransient impulsive effects in a polluted environment. By using the theories of impulsive differential equations, we obtain the globally asymptotically stable condition of a population-extinction solution; we also present the permanent condition for the investigated system. The results indicate that the nontransient and transient impulsive harvesting rate play important roles in system permanence. Finally, numerical analyses are carried out to illustrate the results. Our results provide effective methods for biological resource management in a polluted environment.
\end{abstract}

Keywords: Stage-structured population model; Transient and nontransient impulsive effects; Polluted environment; Permanence

\section{Introduction}

In recent years, the aggravation of environmental pollution not only affects human life style, but also poses a serious threat to the long-term survival of the species. The European Environmental Protection Agency released a new "Health and Environmental Assessment Report" on September 8, 2020, which said that 13 percent of the deaths in 28 European countries are related to environmental pollution. Therefore, the study of the population models in polluted environments is becoming more important. To date, some work has been carried out to study the population models in polluted environments [1-7]. Many workers have adopted a mathematical modeling approach to study the influence of environmental pollution on the survival of biological populations [8-10]. Most of the previous studies assumed that the input of the toxicant was continuous. The toxicants, however, are often emitted to the environment in regular pulses [11]. For example, the spraying of agricultural chemicals can be regarded as time-pulse discharge, though the discharge of the toxin is transient, the influence of the toxin will be long lasting.

Currently, the population system with a stage structure has become another focus of many studies [12-15]. Cai [16] presented a stage-structured single-species model with

(c) The Author(s) 2021. This article is licensed under a Creative Commons Attribution 4.0 International License, which permits use, sharing, adaptation, distribution and reproduction in any medium or format, as long as you give appropriate credit to the original author(s) and the source, provide a link to the Creative Commons licence, and indicate if changes were made. The images or other third party material in this article are included in the article's Creative Commons licence, unless indicated otherwise in a credit line to the material. If material is not included in the article's Creative Commons licence and your intended use is not permitted by statutory regulation or exceeds the permitted use, you will need to obtain permission directly from the copyright holder. To view a copy of this licence, visit http://creativecommons.org/licenses/by/4.0/. 
pulse input in a polluted environment and revealed that a long mature period of the population in a polluted environment can cause it to go extinct. Kang [17] proposed and studied an age-structured population with nonlocal diffusion. Jiao [18] investigated a stagestructured single-population model with nontransient and transient impulsive effects.

\section{The model}

In real life, when facing pollutants from the environment, a mature population and an immature population have different reactions. Considering the population with different stage structures has more practical significance. Inspired by the above discussions, we consider a new stage-structured population model with transient and nontransient impulsive effects in a polluted environment:

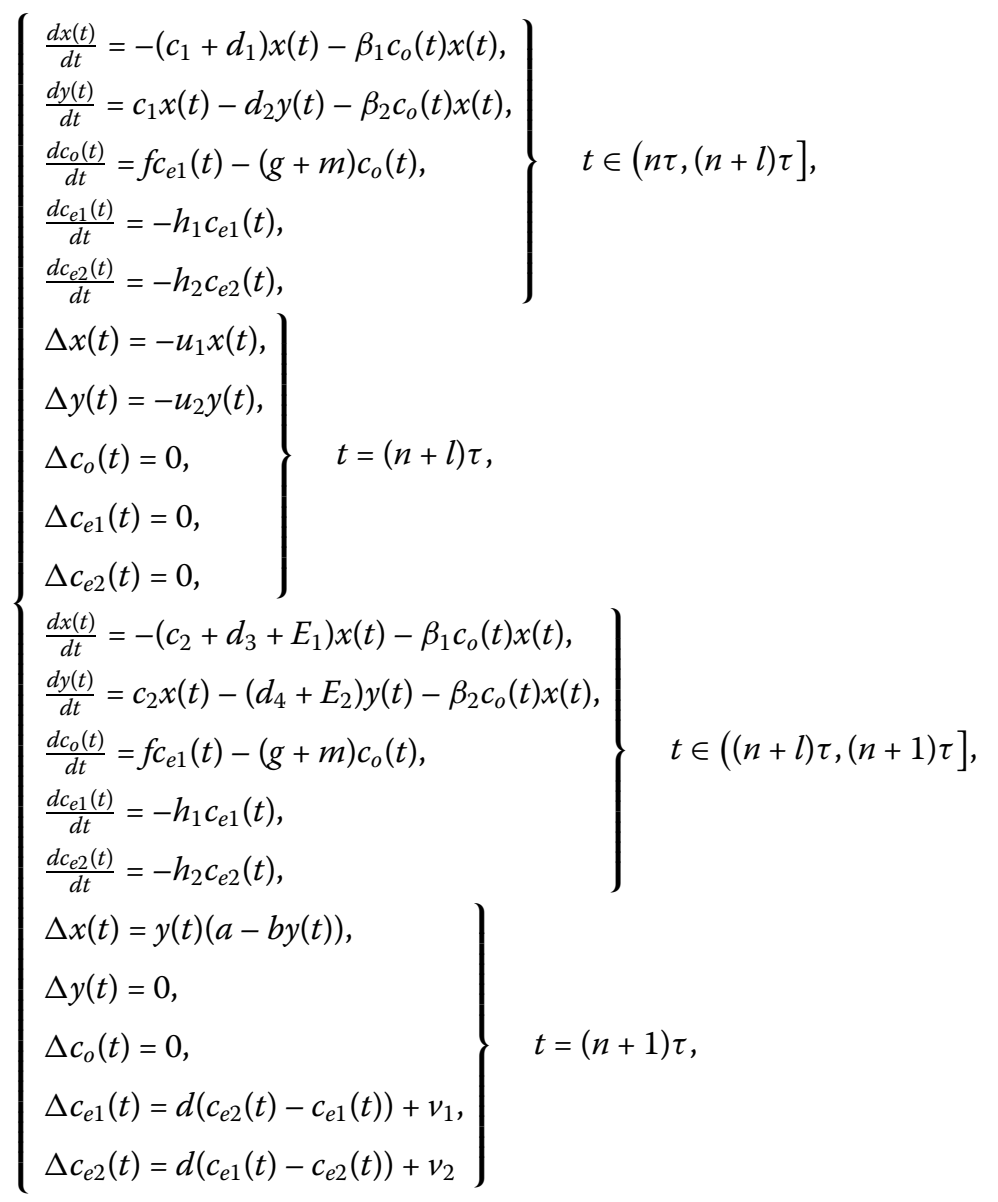

where it is assumed that system (2.1) consists of two lakes that are connected by underground rivers. Environmental toxins will be dispersed between the two lakes due to weather conditions, such as rainy season or flood outbreaks. $x(t), y(t)$ represent the densities of the immature and mature populations, which depend on drinking the water from lake 1 , at time $t$, respectively. $c_{o}(t)$ represents the average concentration of toxins in the organism of the immature and mature populations at time $t . c_{e 1}(t)$ represents the concentration of environmental toxins in lake 1 at time $t . c_{e 2}(t)$ represents the concentration of environmental toxins in lake 2 at time $t . c_{1}>0$ represents the rate of immature population $x$ turning into mature population $y$ on $(n \tau,(n+l) \tau] . d_{1}>0$ represents the natural death 
rate of population $x$ on $(n \tau,(n+l) \tau] . d_{2}>0$ represents the natural death rate of population $y$ on $(n \tau,(n+l) \tau] . \beta_{1}>0$ and $\beta_{2}>0$ represent the mortality coefficient of the immature population and the mature population due to the influence of toxins, respectively. $f>0$ represents the uptake rate of toxin from lake 1 per unit biomass. $g>0$ represents the toxin-consumption coefficient of the population by means of excretion and so on. $m>0$ represents the toxin-consumption coefficient in the population by means of biochemical reactions in the body. $h_{1}>0$ represents the consumption coefficient of the environmental toxins with lake 1 as the water source is affected by processes such as chemical hydrolysis, volatilization, microbial degradation and photosynthesis on $((n+l) \tau,(n+1) \tau] . h_{2}>0$ represents the consumption coefficient of environmental toxins with lake 2 as the water source affected by processes such as chemical hydrolysis, volatilization, microbial degradation and photosynthesis on $((n+l) \tau,(n+1) \tau] .0<u_{1}<1$ represents the transient impulsive harvesting rate of population $x$ at time $t=(n+l) \tau .0<u_{2}<1$ represents the transient impulsive harvesting rate of population $y$ at time $t=(n+l) \tau \cdot c_{2}>0$ represents the rate of immature population $x$ turning into mature population $y$ on $((n+l) \tau,(n+1) \tau] . d_{3}>0$ represents the natural death rate of population $x$ on $((n+l) \tau,(n+1) \tau] . d_{4}>0$ represents the natural death rate of population $y$ on $((n+l) \tau,(n+1) \tau]$. The population is birth pulse as intrinsic rate of nature increase and density dependence rate of the population, which are denoted by $a>0, b>0$, respectively. $E_{1}>0$ represents the nontransient impulsive harvesting rate of the immature population $x$ on $((n+l) \tau,(n+1) \tau] . E_{2}>0$ represents the nontransient impulsive harvesting rate of the mature population $y$ on $((n+l) \tau,(n+1) \tau]$. $0<d<1$ is the dispersal rate between the two lakes. $v_{1}>0$ represents the concentration of toxins that input into lake 1 due to environmental changes at time $t=(n+1) \tau . v_{2}>0$ represents the concentration of toxins that input into lake 2 due to environmental changes at time $t=(n+1) \tau . \tau$ is the period of the population-impulsive harvesting or pulse-input period of toxins.

\section{The dynamics}

Denoting $U(t)=\left(x(t), y(t), c_{o}(t), c_{e 1}(t), c_{e 2}(t)\right)^{T}$, the solution of system (2.1), is a piecewise continuous $U: R_{+} \rightarrow R_{+}^{5}$, where $R_{+}=[0, \infty), R_{+}^{5}=\left\{Z \in R^{5}: U>0\right\}$. $U(t)$ is continuous on $(n \tau,(n+l) \tau]$ and $((n+l) \tau,(n+1) \tau]$. According to Ref. [19], the global existence and uniqueness of the solution of system (2.1) is guaranteed by the smoothness properties of $f_{1}$, which denotes the mapping defined by the right-side of system (2.1).

The subsystem of system (2.1) is

$$
\left\{\begin{array}{l}
\left.\begin{array}{l}
\frac{d x(t)}{d t}=-\left(c_{1}+d_{1}\right) x(t), \\
\frac{d y(t)}{d t}=c_{1} x(t)-d_{2} y(t),
\end{array}\right\} \quad t \in(n \tau,(n+l) \tau], \\
\left.\begin{array}{l}
\Delta x(t)=-u_{1} x(t), \\
\Delta y(t)=-u_{2} y(t),
\end{array}\right\} \quad t=(n+l) \tau, \\
\begin{array}{l}
\frac{d x(t)}{d t}=-\left(c_{2}+d_{3}+E_{1}\right) x(t), \\
\left.\begin{array}{l}
\frac{d y(t)}{d t}=c_{2} x(t)-\left(d_{4}+E_{2}\right) y(t), \\
\Delta x(t)=y(t)(a-b y(t)), \\
\Delta y(t)=0,
\end{array}\right\} \quad t \in((n+l) \tau,(n+1) \tau],
\end{array} \quad t=(n+1) \tau .
\end{array}\right.
$$


Considering the first and second equations and the fifth and sixth equations of system (3.1), we can obtain the analytic solution of system (3.1) between pluses as

$$
\left\{\begin{array}{l}
x(t)=\left\{\begin{array}{l}
x\left(n \tau^{+}\right) \mathrm{e}^{-\left(c_{1}+d_{1}\right)(t-n \tau)}, \quad t \in(n \tau,(n+l) \tau], \\
x\left((n+l) \tau^{+}\right) \mathrm{e}^{-\left(c_{2}+d_{3}+E_{1}\right)(t-(n+l) \tau)}, \quad t \in((n+l) \tau,(n+1) \tau],
\end{array}\right. \\
y(t)=\left\{\begin{array}{c}
\mathrm{e}^{-d_{2}(t-n \tau)}\left[\frac{c_{1}\left(1-\mathrm{e}^{-\left(c_{1}+d_{1}-d_{2}\right)(t-n \tau)}\right)}{c_{1}+d_{1}-d_{2}} x\left(n \tau^{+}\right)+y\left(n \tau^{+}\right)\right], \\
t \in(n \tau,(n+l) \tau], \\
\mathrm{e}^{-\left(d_{4}+E_{2}\right)(t-(n+l) \tau)}\left[\frac{c_{2}\left(1-\mathrm{e}^{-\left(c_{2}+d_{3}+E_{1}-d_{4}-E_{2}\right)(t-(n+l) \tau)}\right)}{c_{2}+d_{3}+E_{1}-d_{4}-E_{2}} x\right. \\
\left.\times\left((n+l) \tau^{+}\right)+y\left((n+l) \tau^{+}\right)\right], \\
t \in((n+l) \tau,(n+1) \tau] .
\end{array}\right.
\end{array}\right.
$$

Considering the third and fourth equations and the seventh and eighth equations of system (3.1), we obtain the stroboscopic map of system (3.1)

$$
\left\{\begin{array}{l}
x\left((n+1) \tau^{+}\right)=(a A+C) x\left(n \tau^{+}\right)+a B y\left(n \tau^{+}\right)-b\left(A x\left(n \tau^{+}\right)+B y\left(n \tau^{+}\right)\right)^{2} \\
y\left((n+1) \tau^{+}\right)=A x\left(n \tau^{+}\right)+B y\left(n \tau^{+}\right)
\end{array}\right.
$$

where

$$
\begin{aligned}
A= & \mathrm{e}^{-\left(d_{4}+E_{2}\right)(1-l) \tau}\left[\frac{c_{2}\left(1-u_{1}\right) \mathrm{e}^{-\left(c_{1}+d_{1}\right) l \tau}\left(1-\mathrm{e}^{-\left(c_{2}+d_{3}+E_{1}-d_{4}-E_{2}\right)(1-l) \tau}\right)}{c_{2}+d_{3}+E_{1}-d_{4}-E_{2}}\right. \\
& +\frac{c_{1}\left(1-u_{2}\right) \mathrm{e}^{-d_{2} l \tau}\left(1-\mathrm{e}^{-\left(c_{1}+d_{1}-d_{2}\right) l \tau}\right)}{c_{1}+d_{1}-d_{2}} \\
B= & \left(1-u_{2}\right) \mathrm{e}^{-\left[d_{2} l \tau+\left(d_{4}+E_{2}\right)(1-l) \tau\right]}<1, \\
C= & \left(1-u_{1}\right) \mathrm{e}^{-\left[\left(c_{1}+d_{1}\right) l \tau+\left(c_{2}+d_{3}+E_{1}\right)(1-l) \tau\right]}<1 .
\end{aligned}
$$

The system (3.3) has two fixed points as $F_{1}(0,0)$ and $F_{2}\left(x^{*}, y^{*}\right)$, where

$$
\left\{\begin{array}{l}
x^{*}=\frac{(1-B)[a A-(1-B)(1-C)]}{b A^{2}}, \quad a A-(1-B)(1-C)>0 \\
y^{*}=\frac{a A-(1-B)(1-C)}{b A}, \quad a A-(1-B)(1-C)>0 .
\end{array}\right.
$$

Theorem 1 (i) If $a A-(1-B)(1-C)<0$, the fixed point $F_{1}(0,0)$ is globally asymptotically stable;

(ii) If $a A-(1-B)(1-C)>0$, the fixed point $F_{2}\left(x^{*}, y^{*}\right)$ is globally asymptotically stable.

Proof For convenience, we make a notation as $\left(x^{n}, y^{n}\right)=\left(x\left(n \tau^{+}\right), y\left(n \tau^{+}\right)\right)$. The linear form of (3.3) can be written as

$$
\left(\begin{array}{l}
x^{n+1} \\
y^{n+1}
\end{array}\right)=J_{1}\left(\begin{array}{l}
x^{n} \\
y^{n}
\end{array}\right)
$$

Obviously, the near dynamics of $F_{1}(0,0)$ and $F_{2}\left(x^{*}, y^{*}\right)$ of $(3.3)$ are determined by the linear system (3.5). The stabilities of the two fixed points of (3.3) are determined by the 
eigenvalues of $J_{1}$ less than 1 . We can determine the eigenvalue of $J_{1}$ less than 1 , if $J_{1}$ satisfies the Jury criteria [20]

$$
1-\operatorname{tr} J_{1}+\operatorname{det} J_{1}>0
$$

(i) If $a A-(1-B)(1-C)<0, F_{1}(0,0)$ is the unique fixed point of (3.3), we have

$$
J_{1}=\left(\begin{array}{cc}
a A+C & a B \\
A & B
\end{array}\right)
$$

\section{Calculating}

$$
\begin{aligned}
1-\operatorname{tr} J_{1}+\operatorname{det} J_{1} & =1-[(a A+C)+B]+[B(a A+C)-a A B] \\
& =1-a A-C-B+B C \\
& =-a A+(1-B)(1-C) \\
& =-[a A-(1-B)(1-C)]>0 .
\end{aligned}
$$

From the Jury criteria, $F_{1}(0,0)$ is locally stable. Then, it is globally asymptotically stable.

(ii) If $a A-(1-B)(1-C)>0, F_{1}(0,0)$ is obviously unstable, $F_{2}\left(x^{*}, y^{*}\right)$ exists, and

$$
J_{1}=\left(\begin{array}{cc}
a A+C-2 b A\left(A x^{*}+B y^{*}\right) & a B-2 b B\left(A x^{*}+B y^{*}\right) \\
A & B
\end{array}\right) .
$$

Calculating

$$
\begin{aligned}
1-\operatorname{tr} J_{1}+\operatorname{det} J_{1}= & 1-\left[a A+C-2 b A\left(A x^{*}+B y^{*}\right)+B\right] \\
& +\left\{B\left[a A+C-2 b A\left(A x^{*}+B y^{*}\right)\right]-A\left[a B-2 b B\left(A x^{*}+B y^{*}\right)\right]\right\} \\
= & 1-a A-C-B+B C+2 b A\left(A x^{*}+B y^{*}\right) \\
= & -[a A+(1-B)(1-C)]+2[a A-(1-B)(1-C)] \\
= & a A-(1-B)(1-C)>0 .
\end{aligned}
$$

From the Jury criteria, $F_{2}\left(x^{*}, y^{*}\right)$ is locally stable. Then, it is globally asymptotically stable. This completes the proof.

According to Theorem 1, and similar to reference [18], the following lemma can be easily proved.

Theorem 2 (i) If aA $-(1-B)(1-C)<0$, the triviality periodic solution $(0,0)$ of system (3.1) is globally asymptotically stable; 
(ii) If aA $-(1-B)(1-C)>0$, the periodic solution $\widetilde{(x(t)}, \widetilde{y(t)})$ of system (3.1) is globally asymptotically stable, where

$$
\left\{\begin{array}{l}
\widetilde{x(t)}=\left\{\begin{array}{l}
x^{*} \mathrm{e}^{-\left(c_{1}+d_{1}\right)(t-n \tau)}, \quad t \in(n \tau,(n+l) \tau], \\
x^{* *} \mathrm{e}^{-\left(c_{2}+d_{3}+E_{1}\right)(t-(n+l) \tau)}, \quad t \in((n+l) \tau,(n+1) \tau],
\end{array}\right. \\
\widetilde{y(t)}=\left\{\begin{array}{l}
\mathrm{e}^{-d_{2}(t-n \tau)}\left[\frac{c_{1}\left(1-\mathrm{e}^{-\left(c_{1}+d_{1}-d_{2}\right)(t-n \tau)}\right)}{c_{1}+d_{1}-d_{2}} x^{*}+y^{*}\right], \quad t \in(n \tau,(n+l) \tau], \\
\mathrm{e}^{-\left(d_{4}+E_{2}\right)(t-(n+l) \tau)}\left[\frac{c_{2}\left(1-\mathrm{e}^{-\left(c_{2}+d_{3}+E_{1}-d_{4}-E_{2}\right)(t-(n+l) \tau)}\right)}{c_{2}+d_{3}+E_{1}-d_{4}-E_{2}} x^{* *}+y^{* *}\right], \\
t \in((n+l) \tau,(n+1) \tau],
\end{array}\right.
\end{array}\right.
$$

and

$$
\left\{\begin{array}{l}
x^{* *}=\left(1-u_{1}\right) \mathrm{e}^{-\left(c_{1}+d_{1}\right) l \tau} x^{*}, \\
y^{* *}=\left(1-u_{2}\right) \mathrm{e}^{-d_{2} l \tau}\left[\frac{c_{1}\left(1-\mathrm{e}^{-\left(c_{1}+d_{1}-d_{2}\right) l \tau}\right)}{c_{1}+d_{1}-d_{2}} x^{*}+y^{*}\right] .
\end{array}\right.
$$

Remark 3 From Theorem 2, For any $\varepsilon>0$, there exists a positive number $t_{0}$, when $t>t_{0}$, we have

$$
\begin{aligned}
& \widetilde{x(t)}-\varepsilon \leq x(t) \leq \widetilde{x(t)}+\varepsilon, \\
& \widetilde{y(t)}-\varepsilon \leq y(t) \leq \widetilde{y(t)}+\varepsilon,
\end{aligned}
$$

then

$$
\begin{aligned}
& m_{1} \leq x(t) \leq M_{1}, \\
& m_{2} \leq y(t) \leq M_{2},
\end{aligned}
$$

where

$$
\begin{aligned}
m_{1}= & {\left[x^{*}+x^{* *}\right]-\varepsilon, } \\
M_{1}= & {\left[x^{*} \mathrm{e}^{-\left(c_{1}+d_{1}\right) l \tau}+x^{* *} \mathrm{e}^{-\left(c_{2}+d_{3}+E_{1}\right)(1-l) \tau}\right]+\varepsilon, } \\
m_{2}= & {\left[y^{*}+y^{* *}\right]-\varepsilon } \\
M_{2}= & \left\{\mathrm{e}^{-d_{2} l \tau}\left[\frac{c_{1}\left(1-\mathrm{e}^{-\left(c_{1}+d_{1}-d_{2}\right) l \tau}\right)}{c_{1}+d_{1}-d_{2}} x^{*}+y^{*}\right]\right. \\
& \left.+\mathrm{e}^{-\left(d_{4}+E_{2}\right)(1-l) \tau}\left[\frac{c_{2}\left(1-\mathrm{e}^{-\left(c_{2}+d_{3}+E_{1}-d_{4}-E_{2}\right)(1-l) \tau}\right)}{c_{2}+d_{3}+E_{1}-d_{4}-E_{2}} x^{* *}+y^{* *}\right]\right\}+\varepsilon .
\end{aligned}
$$

Another subsystem of system (2.1) is also obtained as follows:

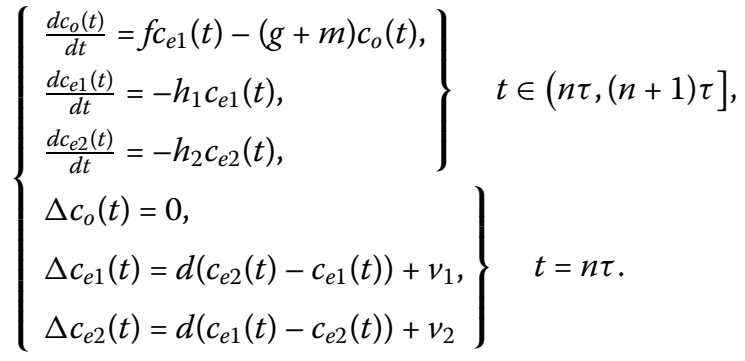


The first, second and third equations of system (3.10) integrate over the interval $(n \tau,(n+$ 1) $\tau$ ], we have

$$
\left\{\begin{aligned}
c_{o}(t) & =\mathrm{e}^{-(g+m)(t-n \tau)}\left[\frac{f\left(1-\mathrm{e}^{-\left(h_{1}-g-m\right)(t-n \tau)}\right)}{h_{1}-g-m} c_{e 1}\left(n \tau^{+}\right)+c_{o}\left(n \tau^{+}\right)\right], \\
t & \in(n \tau,(n+1) \tau], \\
c_{e 1}(t) & =c_{e 1}\left(n \tau^{+}\right) \mathrm{e}^{-h_{1}(t-n \tau)}, \quad t \in(n \tau,(n+1) \tau], \\
c_{e 2}(t) & =c_{e 2}\left(n \tau^{+}\right) \mathrm{e}^{-h_{2}(t-n \tau)}, \quad t \in(n \tau,(n+1) \tau] .
\end{aligned}\right.
$$

Considering the fourth, fifth and sixth equations of system (3.10), the stroboscopic map of system (3.11) is obtained as

$$
\left\{\begin{array}{l}
c_{o}\left((n+1) \tau^{+}\right)=\mathrm{e}^{-(g+m) \tau}\left[\frac{f\left(1-\mathrm{e}^{-\left(h_{1}-g-m\right) \tau}\right)}{h_{1}-g-m} c_{e 1}\left(n \tau^{+}\right)+c_{o}\left(n \tau^{+}\right)\right], \\
c_{e 1}\left((n+1) \tau^{+}\right)=(1-d) c_{e 1}\left(n \tau^{+}\right) \mathrm{e}^{-h_{1} n \tau}+d c_{e 2}\left(n \tau^{+}\right) \mathrm{e}^{-h_{2} n \tau}+v_{1}, \\
c_{e 2}\left((n+1) \tau^{+}\right)=(1-d) c_{e 2}\left(n \tau^{+}\right) \mathrm{e}^{-h_{2} n \tau}+d c_{e 1}\left(n \tau^{+}\right) \mathrm{e}^{-h_{1} n \tau}+v_{2} .
\end{array}\right.
$$

The unique fixed point of (3.12) is obtained as $F\left(c_{o}^{*}, c_{e 1}^{*}, c_{e 2}^{*}\right)$, where

$$
\left\{\begin{array}{l}
c_{o}^{*}=\frac{\mathrm{e}^{-(g+m) \tau} f\left(1-\mathrm{e}^{-\left(h_{1}-g-m\right) \tau}\right) \nu_{2} d \mathrm{e}^{-h_{2} \tau}+\nu_{1}\left[1-(1-d) \mathrm{e}^{-h_{2} \tau}\right]}{\left(h_{1}-g-m\right)\left(1-\mathrm{e}^{-(g+m) \tau}\right)\left[1-(1-d) \mathrm{e}^{-h_{1} \tau}\right]\left(1-\mathrm{v}^{-h_{2} \tau}\right)+d \mathrm{e}^{-h_{2} \tau}\left(1-\mathrm{e}^{-h_{1} \tau}\right)} \\
c_{e 1}^{*}=\frac{v_{2} d \mathrm{e}^{-h_{2} \tau}+v_{1}\left[1-(1-d) \mathrm{e}^{-h_{2} \tau}\right]}{\left[1-(1-d) \mathrm{e}^{-h_{1} \tau}\right]\left(1-\mathrm{e}^{-h_{2} \tau}\right)+d \mathrm{e}^{-h_{2} \tau}\left(1-\mathrm{e}^{-h_{1} \tau}\right)} \\
c_{e 2}^{*}=\frac{v_{1} d \mathrm{e}^{-h_{1} \tau}+v_{2}\left[1-(1-d) \mathrm{e}^{-h_{1} \tau}\right]}{\left[1-(1-d) \mathrm{e}^{-h_{1} \tau}\right]\left(1-\mathrm{e}^{-h_{2} \tau}\right)+d \mathrm{e}^{-h_{2} \tau}\left(1-\mathrm{e}^{-h_{1} \tau}\right)}
\end{array}\right.
$$

Theorem 4 If $d>1 / 2$, the unique fixed point $F\left(c_{o}^{*}, c_{e 1}^{*}, c_{e 2}^{*}\right)$ is globally asymptotically stable.

Proof Making a notation as $\left(c_{o}^{n}, c_{e 1}^{n}, c_{e 2}^{n}\right)=\left(c_{o}\left(n \tau^{+}\right), c_{e 1}\left(n \tau^{+}\right), c_{e 2}\left(n \tau^{+}\right)\right)$, we rewrite the linear form of (3.12) as

$$
\left(\begin{array}{c}
c_{o}^{n+1} \\
c_{e 1}^{n+1} \\
c_{e 2}^{n+1}
\end{array}\right)=J_{2}\left(\begin{array}{c}
c_{o}^{n} \\
c_{e 1}^{n} \\
c_{e 2}^{n}
\end{array}\right)
$$

Obviously, the near dynamics of $F\left(c_{o}^{*}, c_{e 1}^{*}, c_{e 2}^{*}\right)$ is determined by the linear system (3.14). The stabilities of the fixed point of (3.12) are determined by the eigenvalues of $J_{2}$ less than 1. According to the condition of this theorem and $0<\mathrm{e}^{-h_{1} \tau}<1,0<\mathrm{e}^{-h_{2} \tau}<1$, it is easy to obtain the eigenvalues of

$$
J_{2}=\left(\begin{array}{ccc}
\mathrm{e}^{-(g+m) \tau} & \mathrm{e}^{-(g+m) \tau} \frac{f\left(1-\mathrm{e}^{-\left(h_{1}-g-m\right) \tau}\right)}{h_{1}-g-m} & 0 \\
0 & (1-d) \mathrm{e}^{-h_{1} \tau} & d \mathrm{e}^{-h_{2} \tau} \\
0 & d \mathrm{e}^{-h_{1} \tau} & (1-d) \mathrm{e}^{-h_{2} \tau}
\end{array}\right)
$$

which are

$$
\begin{aligned}
& \lambda_{1}=\mathrm{e}^{-(g+m) \tau}<1, \\
& \lambda_{2}=\frac{(1-d)\left(\mathrm{e}^{-h_{1} \tau}+\mathrm{e}^{-h_{2} \tau}\right)+\sqrt{\left[d\left(\mathrm{e}^{-h_{1} \tau}+\mathrm{e}^{-h_{2} \tau}\right)\right]^{2}-4(1-2 d) \mathrm{e}^{-\left(h_{1}+h_{2}\right) \tau}}}{2}
\end{aligned}
$$




$$
\begin{aligned}
< & \frac{(1-d)\left(\mathrm{e}^{-h_{1} \tau}+\mathrm{e}^{-h_{2} \tau}\right)+d\left(\mathrm{e}^{-h_{1} \tau}+\mathrm{e}^{-h_{2} \tau}\right)}{2} \\
& =\frac{\mathrm{e}^{-h_{1} \tau}+\mathrm{e}^{-h_{2} \tau}}{2}<1, \\
\lambda_{3} & =\frac{(1-d)\left(\mathrm{e}^{-h_{1} \tau}+\mathrm{e}^{-h_{2} \tau}\right)-\sqrt{\left[d\left(\mathrm{e}^{-h_{1} \tau}+\mathrm{e}^{-h_{2} \tau}\right)\right]^{2}-4(1-2 d) \mathrm{e}^{-\left(h_{1}+h_{2}\right) \tau}}}{2} \\
& <\frac{(1-d)\left(\mathrm{e}^{-h_{1} \tau}+\mathrm{e}^{-h_{2} \tau}\right)}{2}<1 .
\end{aligned}
$$

Hence, $F\left(c_{o}^{*}, c_{e 1}^{*}, c_{e 2}^{*}\right)$ is locally stable. Then, it is globally asymptotically stable.

Theorem 5 If $d>1 / 2$, the periodic solution $\left(\widetilde{c_{o}(t)}, \widetilde{c_{e 1}(t)}, \widetilde{c_{e 2}(t)}\right)$ of system $(3.10)$ is globally asymptotically stable, where

$$
\left\{\begin{array}{l}
\widetilde{\widetilde{c_{o}(t)}}=\mathrm{e}^{-(g+m)(t-n \tau)}\left[\frac{f\left(1-\mathrm{e}^{-\left(h_{1}-g-m\right)(t-n \tau)}\right)}{h_{1}-g-m} c_{e 1}^{*}+c_{o}^{*}\right], \quad t \in(n \tau,(n+1) \tau], \\
\widetilde{c_{e 1}(t)}=c_{e 1}^{*} \mathrm{e}^{-h_{1}(t-n \tau)}, \quad t \in(n \tau,(n+1) \tau] \\
\widetilde{c_{e 2}(t)}=c_{e 2}^{*} \mathrm{e}^{-h_{2}(t-n \tau)}, \quad t \in(n \tau,(n+1) \tau]
\end{array}\right.
$$

Here, $c_{o}^{*}, c_{e 1}^{*}, c_{e 2}^{*}$ are defined as (3.13).

Remark 6 From Theorem 5, for any $\varepsilon>0$, there exists a positive number $t_{0}$, when $t>t_{0}$, we have

$$
\begin{aligned}
& \widetilde{c_{o}(t)}-\varepsilon \leq c_{o}(t) \leq \widetilde{c_{o}(t)}+\varepsilon, \\
& \widetilde{c_{e 1}(t)}-\varepsilon \leq c_{e 1}(t) \leq \widetilde{c_{e 1}(t)}+\varepsilon, \\
& \widetilde{c_{e 2}(t)}-\varepsilon \leq c_{e 2}(t) \leq \widetilde{c_{e 2}(t)}+\varepsilon,
\end{aligned}
$$

then

$$
\begin{aligned}
& m_{o} \leq c_{o}(t) \leq M_{o}, \\
& m_{e 1} \leq c_{e 1}(t) \leq M_{e 1}, \\
& m_{e 2} \leq c_{e 2}(t) \leq M_{e 2},
\end{aligned}
$$

where

$$
\begin{aligned}
& m_{0}=\frac{f}{h_{1}-g-m} c_{e 1}^{*}+c_{o}^{*}-\varepsilon, \\
& M_{0}=\mathrm{e}^{-(g+m) \tau}\left[\frac{f\left(1-\mathrm{e}^{-\left(h_{1}-g-m\right) \tau}\right)}{h_{1}-g-m} c_{e 1}^{*}+c_{o}^{*}\right]+\varepsilon, \\
& m_{e 1}=c_{e 1}^{*}-\varepsilon \\
& M_{e 1}=c_{e 1}^{*} \mathrm{e}^{-h_{1} \tau}+\varepsilon \\
& m_{e 2}=c_{e 2}^{*}-\varepsilon \\
& M_{e 2}=c_{e 2}^{*} \mathrm{e}^{-h_{2} \tau}+\varepsilon
\end{aligned}
$$


Considering the first and second equations and the eleventh and twelfth equations of system (2.1), we obtain

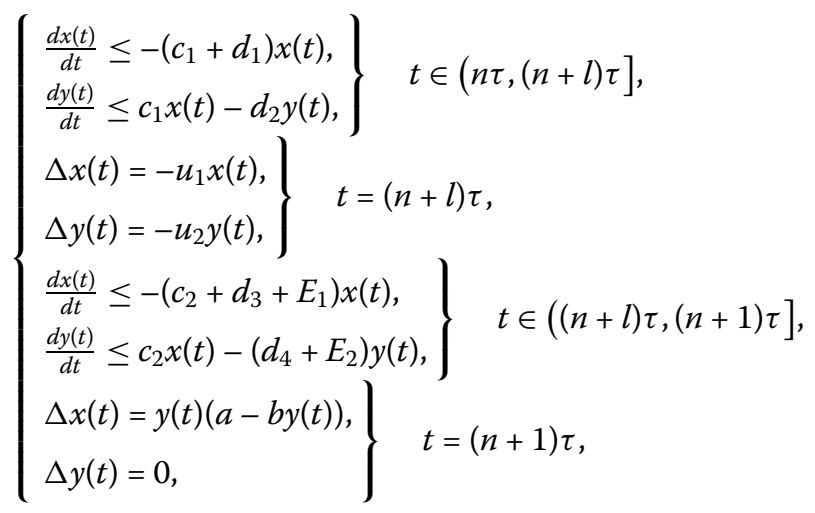

and

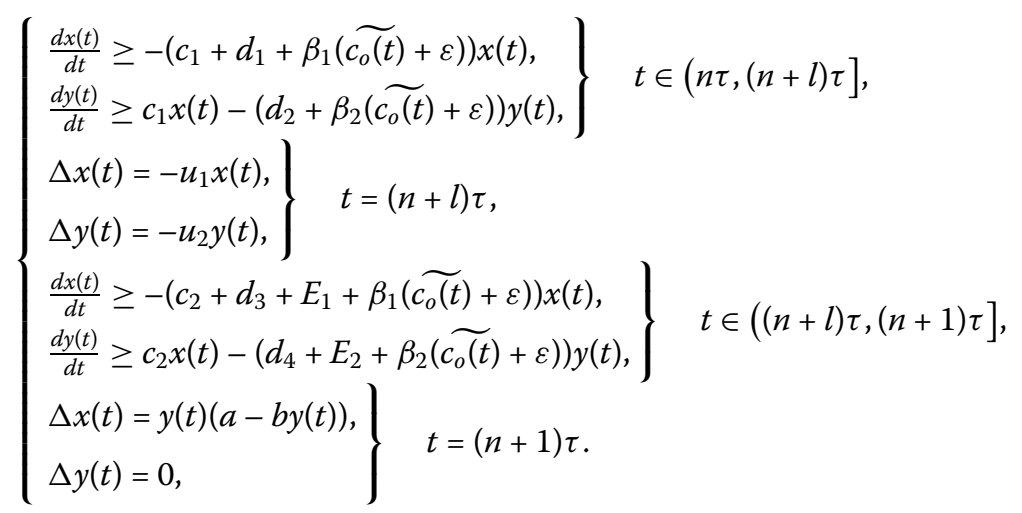

Then, we can obtain the comparative differential equation of system (3.17)

$$
\left\{\begin{array}{l}
\left.\begin{array}{l}
\frac{d x_{1}(t)}{d t}=-\left(c_{1}+d_{1}\right) x_{1}(t), \\
\frac{d y_{1}(t)}{d t}=c_{1} x_{1}(t)-d_{2} y_{1}(t),
\end{array}\right\} \quad t \in(n \tau,(n+l) \tau], \\
\left.\begin{array}{l}
\Delta x_{1}(t)=-u_{1} x_{1}(t), \\
\Delta y_{1}(t)=-u_{2} y_{1}(t),
\end{array}\right\} \quad t=(n+l) \tau, \\
\begin{array}{l}
\frac{d x_{1}(t)}{d t}=-\left(c_{2}+d_{3}+E_{1}\right) x_{1}(t), \\
\frac{d y_{1}(t)}{d t}=c_{2} x_{1}(t)-\left(d_{4}+E_{2}\right) y_{1}(t),
\end{array} \\
\left.\begin{array}{l}
\Delta x_{1}(t)=y_{1}(t)\left(a-b y_{1}(t)\right), \\
\Delta y_{1}(t)=0,
\end{array}\right\} \quad t \in((n+l) \tau,(n+1) \tau],
\end{array}\right.
$$


and the comparative differential equation of system (3.18)

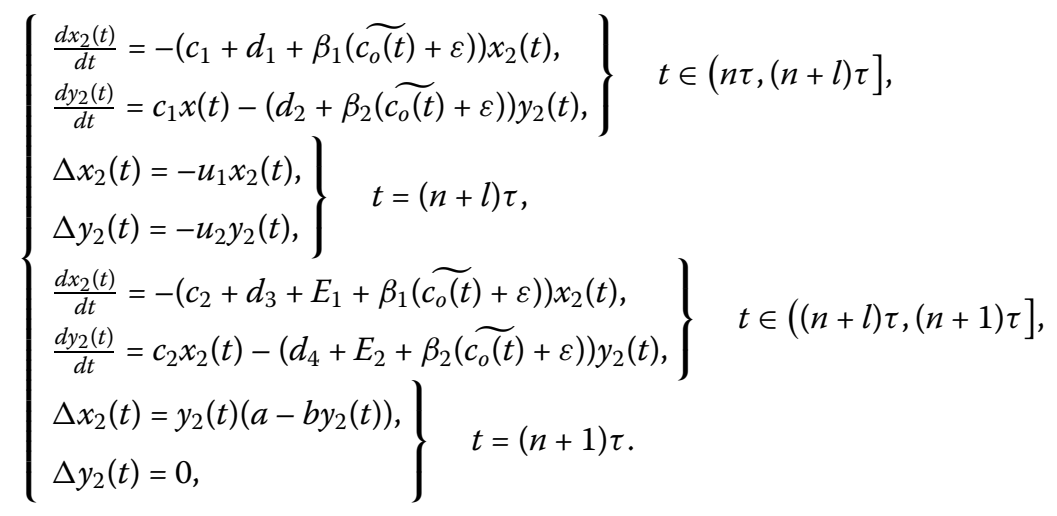

Similarly with Theorem 1 and Theorem 2 , we have:

Theorem 7 (i) If a $A_{1}-\left(1-B_{1}\right)\left(1-C_{1}\right)<0$, the triviality periodic solution of system (3.20) is globally asymptotically stable;

(ii) If a $A_{1}-\left(1-B_{1}\right)\left(1-C_{1}\right)>0$, the triviality periodic solution of system (3.20) is unstable, and the periodic solution $\left(\overline{x_{2}(t)}, \overline{y_{2}(t)}\right)$ of system $(3.20)$ is globally asymptotically stable, where

$$
\left\{\begin{array}{l}
\overline{x_{2}(t)}=\left\{\begin{array}{l}
x_{2}^{*} \mathrm{e}^{-\left(c_{1}+d_{1}+\beta_{1} M_{o}\right)(t-n \tau)}, \quad t \in(n \tau,(n+l) \tau], \\
x_{2}^{* *} \mathrm{e}^{-\left(c_{2}+d_{3}+E_{1}+\beta_{1} M_{o}\right)(t-(n+l) \tau)}, \quad t \in((n+l) \tau,(n+1) \tau],
\end{array}\right. \\
\overline{y_{2}(t)}=\left\{\begin{array}{c}
\mathrm{e}^{-\left(d_{2}+\beta_{2} M_{o}\right)(t-n \tau)}\left[\frac{c_{1}\left(1-\mathrm{e}^{-\left(c_{1}+d_{1}+\beta_{1} M_{o}-d_{2}-\beta_{2} M_{o}\right)(t-n \tau)}\right)}{c_{1}+d_{1}+\beta_{1} M_{o}-d_{2}-\beta_{2} M_{o}} x_{2}^{*}+y_{2}^{*}\right], \\
t \in(n \tau,(n+l) \tau], \\
\mathrm{e}^{-\left(d_{4}+E_{2}+\beta_{2} M_{o}\right)(t-(n+l) \tau)} \\
\times\left[\frac{c_{2}\left(1-\mathrm{e}^{-\left(c_{2}+d_{3}+E_{1}+\beta_{1} M_{o}-d_{4}-E_{2}-\beta_{2} M_{o}\right)(t-(n+l) \tau)}\right.}{c_{2}+d_{3}+E_{1}+\beta_{1} M_{o}-d_{4}-E_{2}-\beta_{2} M_{o}} x_{2}^{* *}+y_{2}^{* *}\right], \\
t \in((n+l) \tau,(n+1) \tau],
\end{array}\right.
\end{array}\right.
$$

Here, $x_{2}^{*}, y_{2}^{*}, x_{2}^{* *}, y_{2}^{* *}$ are defined as follows:

$$
\left\{\begin{array}{l}
x_{2}^{*}=\frac{\left(1-B_{1}\right)\left[a A_{1}-\left(1-B_{1}\right)\left(1-C_{1}\right)\right]}{b A_{1}^{2}}, \quad a A_{1}-\left(1-B_{1}\right)\left(1-C_{1}\right)>0, \\
y_{2}^{*}=\frac{a A_{1}-\left(1-B_{1}\right)\left(1-C_{1}\right)}{b A_{1}}, \quad a A_{1}-\left(1-B_{1}\right)\left(1-C_{1}\right)>0,
\end{array}\right.
$$

and

$$
\left\{\begin{array}{l}
x_{2}^{* *}=\left(1-u_{1}\right) \mathrm{e}^{-\left(c_{1}+d_{1}+\beta_{1} M_{o}\right) l \tau} x_{2}^{*}, \\
y_{2}^{* *}=\left(1-u_{2}\right) \mathrm{e}^{-\left(d_{2}+\beta_{2} M_{o}\right) l \tau}\left[\frac{c_{1}\left(1-\mathrm{e}^{-\left(c_{1}+d_{1}+\beta_{1} M_{o}-d_{2}-\beta_{2} M_{o}\right) l \tau}\right)}{c_{1}+d_{1}+\beta_{1} M_{o}-d_{2}-\beta_{2} M_{o}} x_{2}^{*}+y_{2}^{*}\right] .
\end{array},\right.
$$

where

$$
\begin{aligned}
A_{1}= & \mathrm{e}^{-\left(d_{4}+E_{2}+\beta_{2} M_{o}\right)(1-l) \tau}\left[\frac{c_{2}\left(1-u_{1}\right) \mathrm{e}^{-\left(c_{1}+d_{1}+\beta_{1} M_{o}\right) l \tau}\left(1-\mathrm{e}^{-\left(c_{2}+d_{3}+E_{1}+\beta_{1} M_{o}-d_{4}-E_{2}-\beta_{2} M_{o}\right)(1-l) \tau}\right)}{c_{2}+d_{3}+E_{1}+\beta_{1} M_{o}-d_{4}-E_{2}-\beta_{2} M_{o}}\right. \\
& \left.+\frac{c_{1}\left(1-u_{2}\right) \mathrm{e}^{-\left(d_{2}+\beta_{2} M_{o}\right) l \tau}\left(1-\mathrm{e}^{-\left(c_{1}+d_{1}+\beta_{1} M_{o}-d_{2}-\beta_{2} M_{o}\right) l \tau}\right)}{c_{1}+d_{1}+\beta_{1} M_{o}-d_{2}-\beta_{2} M_{o}}\right],
\end{aligned}
$$




$$
\begin{aligned}
& B_{1}=\left(1-u_{2}\right) \mathrm{e}^{-\left[\left(d_{2}+\beta_{2} M_{o}\right) l \tau+\left(c_{1}+d_{1}+\beta_{1} M_{o}-d_{2}-\beta_{2} M_{o}\right)(1-l) \tau\right]}<1, \\
& C_{1}=\left(1-u_{1}\right) \mathrm{e}^{-\left[\left(c_{1}+d_{1}+\beta_{1} M_{o}\right) l \tau+\left(c_{2}+d_{3}+E_{1}+\beta_{1} M_{o}\right)(1-l) \tau\right]}<1 .
\end{aligned}
$$

Remark 8 From Theorem 7, For any $\varepsilon>0$, there exists a positive number $t_{0}$, such that for $t>t_{0}$,

$$
\begin{aligned}
& \overline{x_{2}(t)}-\varepsilon \leq x_{2}(t) \leq \overline{x_{2}(t)}+\varepsilon, \\
& \overline{y_{2}(t)}-\varepsilon \leq y_{2}(t) \leq \overline{y_{2}(t)}+\varepsilon,
\end{aligned}
$$

then

$$
\begin{aligned}
& m_{21} \leq x_{2}(t) \leq M_{21}, \\
& m_{22} \leq y_{2}(t) \leq M_{22},
\end{aligned}
$$

where

$$
\begin{aligned}
m_{21}= & {\left[x_{2}^{*}+x_{2}^{* *}\right]-\varepsilon, } \\
M_{21}= & {\left[x_{2}^{*} \mathrm{e}^{-\left(c_{1}+d_{1}+\beta_{1} M_{o}\right) l \tau}+x_{2}^{* *} \mathrm{e}^{-\left(c_{2}+d_{3}+E_{1}+\beta_{1} M_{o}\right)(1-l) \tau}\right]+\varepsilon } \\
m_{22}= & {\left[y_{2}^{*}+y_{2}^{* *}\right]-\varepsilon } \\
M_{22}= & \mathrm{e}^{-\left(d_{2}+\beta_{2} M_{o}\right) l \tau}\left[\frac{c_{1}\left(1-\mathrm{e}^{-\left(c_{1}+d_{1}+\beta_{1} M_{o}-d_{2}-\beta_{2} M_{o}\right) l \tau}\right)}{c_{1}+d_{1}+\beta_{1} M_{o}-d_{2}-\beta_{2} M_{o}} x_{2}^{*}+y_{2}^{*}\right] \\
& +\mathrm{e}^{-\left(d_{4}+E_{2}+\beta_{2} M_{o}\right)(1-l) \tau}\left[\frac{c_{2}\left(1-\mathrm{e}^{-\left(c_{2}+d_{3}+E_{1}+\beta_{1} M_{o}-d_{4}-E_{2}-\beta_{2} M_{o}\right)(1-l) \tau}\right)}{c_{2}+d_{3}+E_{1}+\beta_{1} M_{o}-d_{4}-E_{2}-\beta_{2} M_{o}} x_{2}^{* *}+y_{2}^{* *}\right]+\varepsilon .
\end{aligned}
$$

From the above theorems and remarks, we present an important theorem in this paper.

Theorem 9 (i) aA $-(1-B)(1-C)<0$, the population $x(t)$ and $y(t)$ go extinct;

(ii) If $a A_{1}-\left(1-B_{1}\right)\left(1-C_{1}\right)>0$, the system is permanent.

Proof (i) In the condition of $a A-(1-B)(1-C)<0$, the trivial periodic solution is globally asymptotically stable, that is when $t \rightarrow \infty$, we have $x(t) \rightarrow 0$ and $y(t) \rightarrow 0$. According to (3.17), (3.19) and comparison with the theorem of the impulsive equation [21], we know that $0 \leq x(t) \leq x_{1}(t)=x(t)$ and $0 \leq y(t) \leq y_{1}(t)=y(t)$. These show that the populations $x(t)$ and $y(t)$ go extinct.

(ii) By the condition $a A_{1}-\left(1-B_{1}\right)\left(1-C_{1}\right)>0$, it is easy to show that $a A-(1-B)(1-C)>$ 0 . According to (18)-(20) and with the comparison theorem of the impulsive equation [21], we can obtain that $\overline{x_{2}(t)}-\varepsilon \leq x_{2}(t) \leq x(t), x(t) \leq x_{1}(t) \leq \widetilde{x(t)}+\varepsilon$. From Remark 3 and Remark 8, we have $m_{21} \leq x_{2}(t), x_{1}(t) \leq M_{1}$, then $m_{21} \leq x(t) \leq M_{1}$. Similarly, $m_{22} \leq y(t) \leq$ $M_{2}$, From Remark 6, we have $m_{o} \leq c_{o}(t) \leq M_{o}, m_{e 1} \leq c_{e 1}(t) \leq M_{e 1}, m_{e 2} \leq c_{e 2}(t) \leq M_{e 2}$. This completes the proof.

\section{Numerical simulations}

Using numerical simulations, we analyze the influences of $E_{1}$ and $u_{1}$ on system (2.1). If it is assumed that $x(t)=1, y(t)=0.5, c_{o}(t)=0.5, c_{e 1}(t)=0.4, c_{1}=0.1, d_{1}=0.1, \beta_{1}=0.01$, 
(a)
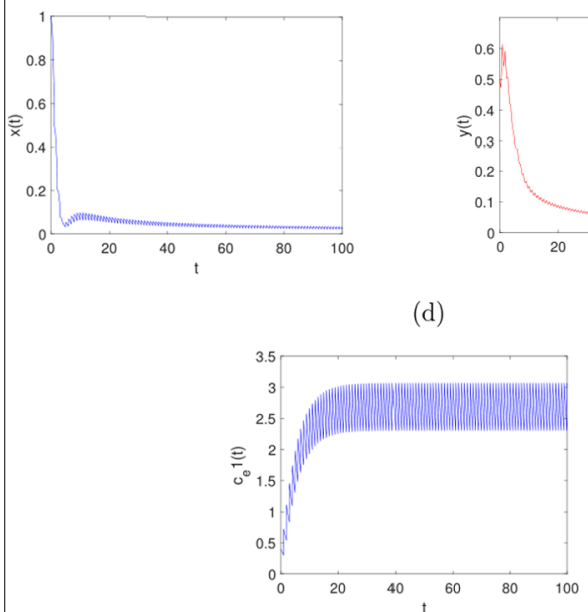

(b)

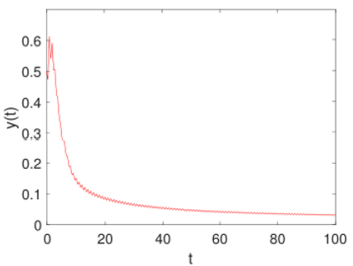

(c)

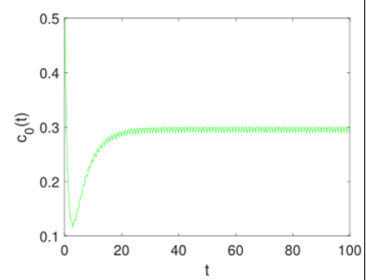

(e)

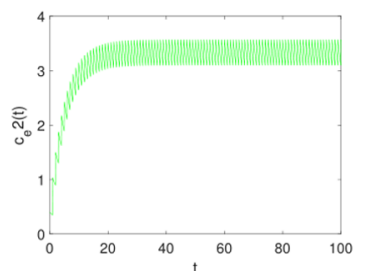

Figure 1 The permanence of system (2.1) with $x(t)=1, y(t)=0.5, c_{0}(t)=0.5, c_{e 1}(t)=0.4, c_{1}=0.1, d_{1}=0.1$, $\beta_{1}=0.01, d_{2}=0.3, \beta_{2}=0.01, f=0.1, g=0.4, m=0.5, h_{1}=0.3, h_{2}=0.15, a=0.4, b=1, c_{2}=0.5, d_{3}=0.1$, $E_{1}=0.08, d_{4}=0.1, E_{2}=0.05, u_{1}=0.01, u_{2}=0.03, d=0.5, v_{1}=0.4, v_{2}=0.5, I=0.5, \tau=1 ;(\mathbf{a})$ time series of $x(t)$; (b) time series of $y(t) ;(\mathbf{c})$ time series of $c_{0}(t) ;(\mathbf{d})$ time series of $c_{e 1}(t) ;(\mathbf{e})$ time series of $c_{e 2}(t)$

$d_{2}=0.3, \beta_{2}=0.01, f=0.1, g=0.4, m=0.5, h_{1}=0.3, h_{2}=0.15, a=0.4, b=1, c_{2}=0.5$, $d_{3}=0.1, E_{1}=0.08, d_{4}=0.1, E_{2}=0.05, u_{1}=0.01, u_{2}=0.03, d=0.5, v_{1}=0.4, v_{2}=0.5, l=0.5$, $\tau=1$, the system is permanent, as shown in Fig. 1 .

\subsection{The simulation of system (2.1) affected by parameter $E_{1}$}

Assuming that $x(t)=1, y(t)=0.5, c_{0}(t)=0.5, c_{e 1}(t)=0.4, c_{1}=0.1, d_{1}=0.1, \beta_{1}=0.01, d_{2}=$ $0.3, \beta_{2}=0.01, f=0.1, g=0.4, m=0.5, h_{1}=0.3, h_{2}=0.15, a=0.4, b=1, c_{2}=0.5, d_{3}=0.1$, $E_{1}=0.3, d_{4}=0.1, E_{2}=0.05, u_{1}=0.01, u_{2}=0.03, d=0.5, v_{1}=0.4, v_{2}=0.5, l=0.5, \tau=1$, the population-extinction periodic solution $(0,0)$ of system $(2.1)$ is globally asymptotically stable, as shown in Fig. 2. From Figs. 1 and 2, if all parameters of system (2.1) are fixed, when $E_{1}=0.08$, we can obtain $a A-(1-B)(1-C)=0.0120>0$, then the condition of Theorem 9(ii) is satisfied, and the system is permanent. When $E_{1}=0.3$, we can obtain $a A-(1-B)(1-C)=-0.0108<0$, then the condition of Theorem 9(i)is satisfied, and the populations $x(t)$ and $y(t)$ go extinct.

\subsection{The simulation of system (2.1) affected by parameter $u_{1}$}

Assuming that $x(t)=1, y(t)=0.5, c_{o}(t)=0.5, c_{e 1}(t)=0.4, c_{1}=0.1, d_{1}=0.1, \beta_{1}=0.01, d_{2}=$ $0.3, \beta_{2}=0.01, f=0.1, g=0.4, m=0.5, h_{1}=0.3, h_{2}=0.15, a=0.4, b=1, c_{2}=0.5, d_{3}=0.1$, $E_{1}=0.08, d_{4}=0.1, E_{2}=0.05, u_{1}=0.2, u_{2}=0.03, d=0.5, v_{1}=0.4, v_{2}=0.5, l=0.5, \tau=1$, the population-extinction periodic solution $(0,0)$ of system $(2.1)$ is globally asymptotically stable, as shown in Fig. 3. From Figs. 1 and 3, if all parameters of system (2.1) are fixed, when $u_{1}=0.01$, we can obtain $a A-(1-B)(1-C)=0.0120>0$, then the condition of Theorem 9(ii) is satisfied, and the system is permanent. When $u_{1}=0.2$, we can obtain $a A-(1-B)(1-C)=-0.0035<0$, then the condition of Theorem 9(i) is satisfied, and the populations $x(t)$ and $y(t)$ go extinct. 
(a)
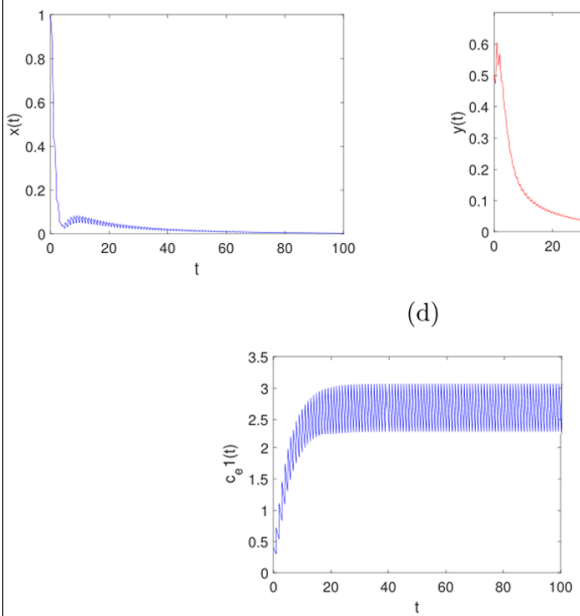

(b)

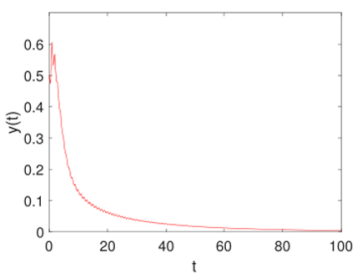

(c)

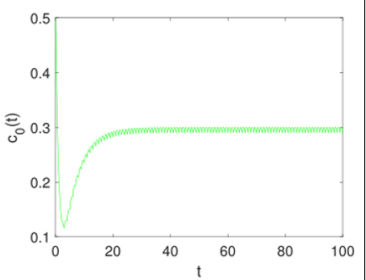

(e)

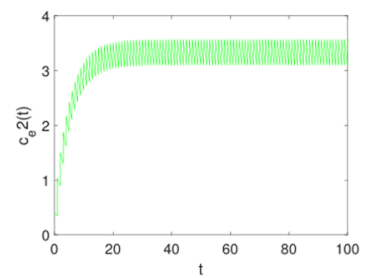

Figure 2 Globally asymptotically stable population-extinction periodic solution of system (2.1) with $x(t)=1$, $y(t)=0.5, c_{0}(t)=0.5, c_{e 1}(t)=0.4, c_{1}=0.1, d_{1}=0.1, \beta_{1}=0.01, d_{2}=0.3, \beta_{2}=0.01, f=0.1, g=0.4, m=0.5$, $h_{1}=0.3, h_{2}=0.15, a=0.4, b=1, c_{2}=0.5, d_{3}=0.1, E_{1}=0.3, d_{4}=0.1, E_{2}=0.05, u_{1}=0.01, u_{2}=0.03, d=0.5$, $v_{1}=0.4, v_{2}=0.5, I=0.5, \tau=1 ;(\mathbf{a})$ time series of $x(t) ;(\mathbf{b})$ time series of $y(t) ;(\mathbf{c})$ time series of $c_{0}(t) ;(\mathbf{d})$ time series of $C_{e 1}(t) ;(\mathbf{e})$ time series of $c_{e 2}(t)$

(a)

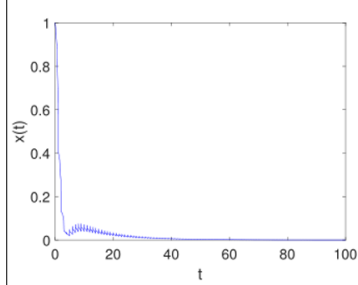

(d)

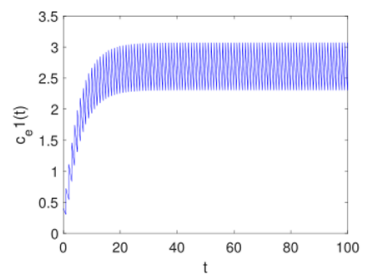

(b)

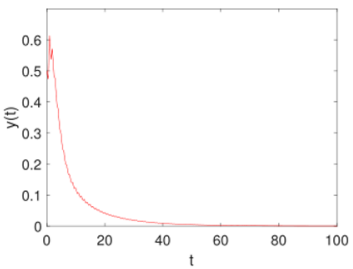

(e)

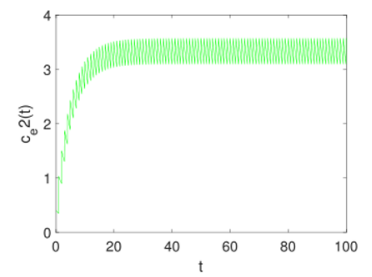

Figure 3 Globally asymptotically stable population-extinction periodic solution of system (2.1) with $x(t)=1$, $y(t)=0.5, c_{0}(t)=0.5, c_{e 1}(t)=0.4, c_{1}=0.1, d_{1}=0.1, \beta_{1}=0.01, d_{2}=0.3, \beta_{2}=0.01, f=0.1, g=0.4, m=0.5$ $h_{1}=0.3, h_{2}=0.15, a=0.4, b=1, c_{2}=0.5, d_{3}=0.1, E_{1}=0.08, d_{4}=0.1, E_{2}=0.05, u_{1}=0.2, u_{2}=0.03, d=0.5$, $v_{1}=0.4, v_{2}=0.5, I=0.5, \tau=1 ;(\mathbf{a})$ time series of $x(t) ;(\mathbf{b})$ time series of $y(t) ;(\mathbf{c})$ time series of $c_{0}(t) ;(\mathbf{d})$ time series of $c_{e 1}(t) ;(\mathbf{e})$ time series of $c_{e 2}(t)$

\section{Discussion}

In this paper, we propose a new stage-structured population model with impulsive effects in a polluted environment. The condition for the globally asymptotic stability of the triviality periodic solution $(0,0)$ of system $(2.1)$ is obtained, and the permanent condition of system (2.1) is also obtained. It can be seen from the analyses that the nontransient harvesting rate and transient impulsive harvesting rate play important roles in system (2.1). 
From the numerical simulation of Figs. 1 and 2, we can deduce that there must exist a nontransient impulsive harvesting population threshold $E_{1}^{*}$, which satisfies $0.08<E_{1}^{*}<0.3$. If $E_{1}<E_{1}^{*}$, the system (2.1) is permanent, and if $E_{1}>E_{1}^{*}$, the populations go extinct. From the numerical simulation of Figs. 1 and 3, we can also deduce that there exists a transient impulsive harvesting population threshold $u_{1}^{*}$, which satisfies $0.01<u_{1}^{*}<0.2$. If $u_{1}<u_{1}^{*}$, the system (2.1) is permanent, and if $u_{1}>u_{1}^{*}$, the populations go extinct. Comparing the figures, it is obvious that under the condition of system persistence, the nontransient impulsive harvesting rate $E_{1}$ and the transient impulsive harvesting rate $u_{1}$ are both smaller than under the condition of population extinction. Hence, we can protect biological diversity by reducing the amount of transient pulse harvest, or reducing the nontransient impulsive harvesting intensity.

Acknowledgements

The authors thank the editor and anonymous referees for useful comments that led to a great improvement of the paper.

\section{Funding}

This paper was supported by the National Natural Science Foundation of China $(11761019,11361014)$, the Graduate Education Innovation Program of Guizhou Province (QJH- YJSCXJH [2019] 065), the Science Technology Foundation of Guizhou Province (20175736-001, 2008038), the Project of High Level Creative Talents in Guizhou Province (No. 20164035), and the Major Research Projects on Innovative Groups in Guizhou Provincial Education Department (No. [2018]019).

Availability of data and materials

Data sharing is not applicable to this article as no datasets were generated or analyzed during the current study.

\section{Declarations}

Competing interests

The authors declare that they have no competing interests.

\section{Authors' contributions}

All authors read and approved the final manuscript.

\section{Publisher's Note}

Springer Nature remains neutral with regard to jurisdictional claims in published maps and institutional affiliations.

Received: 19 October 2020 Accepted: 27 September 2021 Published online: 23 December 2021

\section{References}

1. Cai, S., Jiao, J., Li, L.: Dynamics of a single population system with impulsively unilateral diffusion and imp., input toxins in polluted environment. Adv. Differ. Equ. 1, 1-15 (2015)

2. Li, D., Guo, T., Xu, Y.: The effects of impulsive toxicant input on a single-species population in a small polluted environment. Math. Biosci. Eng. 16, 8179-8194 (2019)

3. Liu, G., Meng, X.: Optimal harvesting strategy for a stochastic mutualism system in a polluted environment with regime switching. Phys. A, Stat. Mech. Appl. 536, 432-449 (2019)

4. Li, Z., Shuai, Z., Wang, K.: Persistence and extinction of single population in a polluted environment. Electron. J. Differ. Equ. 108, 1 (2004)

5. Lan, G., Wei, C., Zhang, S.: Long time behaviors of single-species population models with psychological effect and impulsive toxicant in polluted environments. Phys. A, Stat. Mech. Appl. 521, 828-842 (2019)

6. Zhao, Y., Yuan, S., Ma, J.: Survival and stationary distribution analysis of a stochastic competitive model of three species in a polluted environment. Bull. Math. Biol. 77, 1285-1326 (2015)

7. Tuong, T.: Epidemic SIS model in air-polluted environment. J. Appl. Math. Comput. 64, 53-69 (2020)

8. He, X., Shan, M., Liu, M.: Persistence and extinction of an $n$-species mutualism model with random perturbations in a polluted environment. Phys. A, Stat. Mech. Appl. 419, 313-324 (2018)

9. Guo, S.: Survival analysis of a stochastic cooperation system with functional response in a polluted environment. Adv. Differ. Equ. 1, 291-304 (2020)

10. Jiao, J., Cai, S., Chen, L.: Dynamics of the genic mutational rate on a population system with birth pulse and impulsive input toxins in polluted environment. J. Appl. Math. Comput. 40, 445-457 (2012)

11. Liu, B., Chen, L., Zhang, Y.: The effects of impulsive toxicant input on a population in a polluted environment. J. Biol. Syst. 11, 265-274 (2003)

12. Jiao, J., Yang, X., Chen, L.: Harvesting on a stage-structured single population model with mature individuals in a polluted environment and pulse input of environmental toxin. Biosystems 97, 186-190 (2009)

13. Jiao, J., Cai, S., Chen, L.: Analysis of a stage-structured predator-prey system with birth pulse and impulsive harvesting at different moments. Nonlinear Anal., Real World Appl. 12, 2232-2244 (2011) 
14. Jiao, J., et al.: Dynamics of a stage-structured predator-prey model with prey impulsively diffusing between two patches. Nonlinear Anal., Real World Appl. 11, 2748-2756 (2010)

15. Luo, Z:: Optimal control for an age-dependent predator-prey system in a polluted environment. J. Appl. Math Comput. 44, 491-509 (2014)

16. Cai, S.: A stage-structured single species model with pulse input in a polluted environment. Nonlinear Dyn. 57, 375-382 (2009)

17. Kang, H., Ruan, S., Yu, X.: Age-structured population dynamics with nonlocal diffusion. J. Differ. Equ. (2020). https://doi.org/10.1007/s10884-020-09860-5

18. Jiao, J., et al.: Threshold dynamics of a stage-structured single population model with non-transient and transient impulsive effects. Appl. Math. Lett. 97, 88-92 (2019)

19. Lakshmikantham, V.: Theory of Impulsive Differential Equations. World Scientific, Singapore (1989)

20. Jury, E.: Inners and Stability of Dynamic Systems. Wiley, New York (1974)

21. Song, X., Chen, L.: Uniform persistence and global attractivity for nonautonomous competitive systems with dispersion. J. Syst. Sci. Complex. 3, 307-314 (2002)

Submit your manuscript to a SpringerOpen ${ }^{\circ}$ journal and benefit from:

- Convenient online submission

- Rigorous peer review

Open access: articles freely available online

- High visibility within the field

- Retaining the copyright to your article

Submit your next manuscript at $\boldsymbol{\nabla}$ springeropen.com 